\title{
A study of normal nerve action potentials using an averaging technique (barrier grid storage tube)
}

\author{
R. W. Gilliatt, I. D. Melville, ${ }^{1}$ A. S. Velate, AND R. G. Willison
}

From the Institute of Neurology, Queen Square, London

When human nerve action potentials were first recorded by Dawson and Scott (1949), the median or ulnar nerve was stimulated at the wrist and the action potential of the ascending volley recorded by means of surface electrodes placed over the course of the nerve in the upper arm. The ascending volley contained antidromic impulses in motor fibres as well as orthodromic impulses in sensory fibres, and in order to eliminate the contribution of motor fibres, Dawson (1956) introduced the technique of stimulating digital nerve fibres in the finger, the recording electrodes being placed over the median or ulnar nerve in the arm. Subsequently it was shown by Sears (1959) that it is also possible to stimulate the median or ulnar nerve at the wrist and to record the action potential of the volley passing antidromically in sensory fibres in the finger.

These techniques were soon applied to the study of patients with peripheral nerve lesions (Gilliatt and Sears, 1958; Downie and Newell, 1961 ; Campbell, 1962) but, in spite of their widespread use, certain fundamental questions have not been answered. For example, to what extent do the position and separation of recording electrodes influence nerve action potential amplitude and waveform? Does a stimulus through a cathode on the skin excite the nerve under the cathode? Is it permissible to estimate conduction velocity directly from the conduction time between the stimulus and the onset of a response? Alternatively, should two sets of recording electrodes at different distances from the stimulating cathode be used, the shorter conduction time being subtracted from the longer in order to estimate velocity?

One difficulty which has always arisen in the study of such problems has been the small size of nerve action potentials when recorded through the skin. However, the development of the barrier grid storage tube now makes it possible to obtain clear records of nerve action potentials even when these are of lower voltage than the noise level of the amplifiers (Buller and Styles, 1959; 1961). In the present work we have therefore used a barrier grid storage tube to study

\footnotetext{
${ }^{1}$ Clinical research fellow, M.R.C.
}

nerve action potentials in healthy subjects, and in particular to establish the extent to which such potentials may be affected by slight variations in technique.

\section{METHODS}

Subjects were examined on a couch in a warm room. To ensure that the subject was warm at the beginning of an experiment, he sat with both arms in hot water for five minutes beforehand; he was then covered with a blanket, and the arm to be examined was wrapped in cotton wool. Provided that these precautions against cooling were taken, the skin temperature of the arm and hand under examination remained above $35^{\circ} \mathrm{C}$. in all experiments.

The stimulus, which was set to repeat once or twice per second, was a condenser discharge (time-constant $100 \mu \mathrm{sec}$.) delivered through a 1-1 isolating transformer, its voltage being continuously variable up to 300 volts; the output impedance was not greater than 500 ohms.

For stimulation of digital nerve fibres, silver ring electrodes covered with lint soaked in saline, as described by Dawsol (1956), were used for both cathode and anode. When stimuli were delivered to the median or ulnar nerve at the wrist the cathode was a Walter-type E.E.G. electrode also covered with lint soaked in saline, its diameter being approximately $1 \mathrm{~cm}$. Except in a few experiments specifically mentioned in the text, the anode was a metal plate, $3.5 \times 6.0 \mathrm{~cm}$., attached to the dorsum of the wrist.

Nerve action potentials were recorded from the skin through either Walter-type E.E.G. electrodes or the saddle-shaped electrodes described by Dawson and Scott (1949), the silver electrodes being covered with lint soaked in saline in both cases. Electrodes were mounted in pairs on Perspex plates which could be lightly strapped to the arm, the inter-electrode distance being $4.0 \mathrm{~cm}$. unless otherwise stated.

When recordings were made from the electrodes at the wrist, the subject was earthed through a metal plate on the dorsum of the hand. For recording at the elbow the earth plate was placed on the flexor aspect of the forearm.

Nerve action potentials were amplified and displayed on one beam of a cathode ray tube, the other beam being used to provide a time scale. A digital delay and timescale generator (Pitman, 1958) made it possible to trigger the stimulus and the sweep independently. It was there- 
fore possible to delay the onset of the sweep by any interval required, and thus to display nerve action potentials on an expanded time base, the stimulus remaining locked to a fixed point on the time-scale before the onset of the sweep. A voltage clamp was released $1.0 \mathrm{msec}$. before the start of the sweep to ensure that the baseline was not displaced by stimulus artefact or random background activity.

In previous studies the traces were superimposed photographically but in the present work sweeps were fed into a response averager incorporating a barrier grid storage tube. One hundred sweeps were stored and the average of these was displayed on a cathode ray tube and photographed. For each nerve action potential to be recorded, the averaging process was usually repeated three times, the three averaged responses (each based on 100 sweeps) being superimposed; an averaged time scale derived from a crystal-controlled oscillator was then photographed below the traces. Records were made on $70 \mathrm{~mm}$. recording paper. For subsequent measurement a ruled cursor and magnifying lens were used, and time intervals were measured to the nearest $0.05 \mathrm{msec}$.

A brief description of the response averager is given in the Appendix.

\section{RESULTS}

POSITION AND SEPARATION OF RECORDING ELECTRODES In their original experiments Dawson and Scott (1949) used a pair of recording electrodes placed on the skin over the course of the median or ulnar nerve; thus an ascending volley of impulses passed under each electrode in turn. Subsequently, Gilliatt and Willison (1963) used a single electrode over the nerve to record the potential changes relative to a remote electrode on the opposite side of the limb. These authors commented that the original technique of Dawson and Scott was the easier of the two, particularly in subjects who did not relax well, since the electrical activity of adjacent muscles tended to affect each electrode to a similar extent and therefore to be rejected by the differential amplifier. This resulted in a stable baseline when recordings were being made at high amplification, whereas with widely separated electrodes muscle activity proved more troublesome to eliminate. Most clinical workers have followed Dawson and Scott in using a pair of recording electrodes placed over the nerve, but little attention has been paid to the question of interelectrode distance and its effect on action potential amplitude and waveform. This problem has therefore been re-investigated.

Near-maximal stimuli were delivered to the median nerve at the wrist, stimulus intensity remaining constant throughout each experiment. Five recording electrodes were placed over the course of the median nerve in the arm, the distal electrode being at the level of the elbow and the others at approximately $3 \mathrm{~cm}$. intervals proximal to it. A

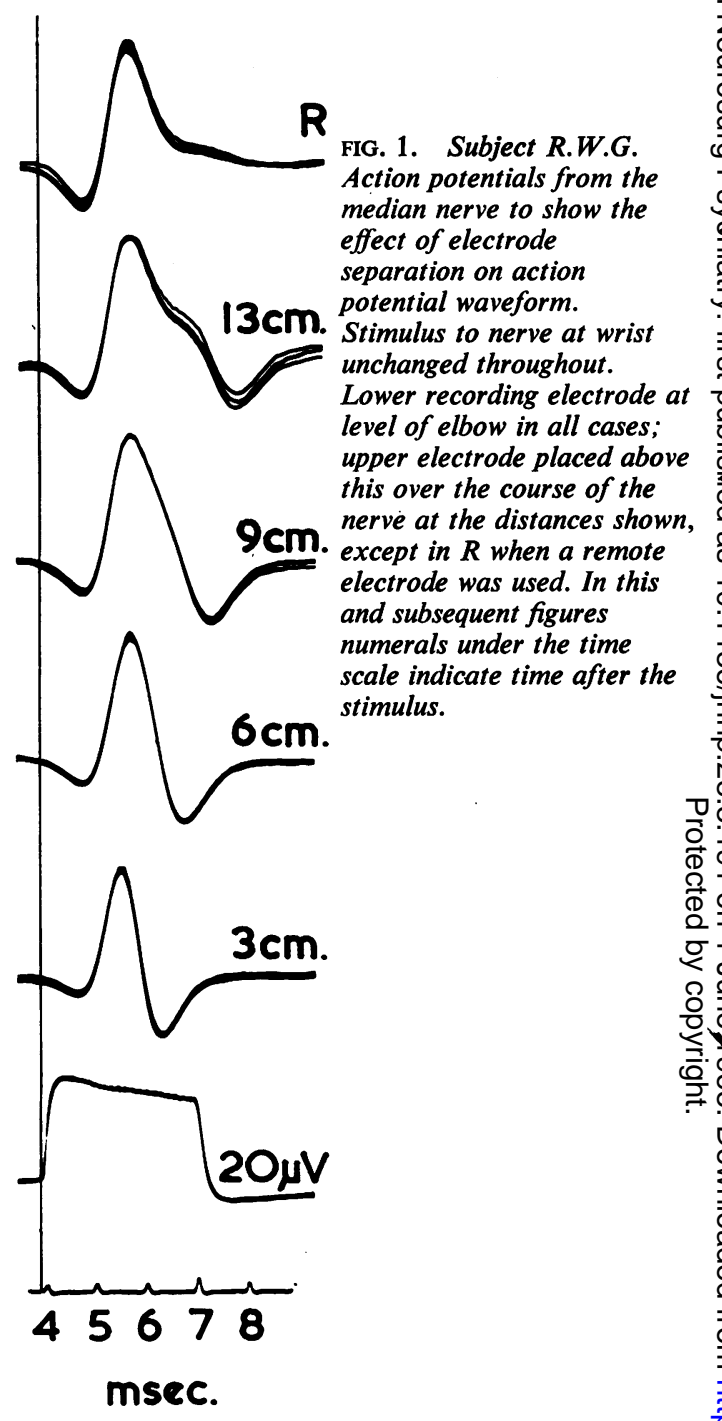

sixth remote electrode was placed over the deltoid insertion on the lateral side of the arm. Recordings were then made between the distal electrode and each of the others in turn.

A typical result is shown in Fig. 1, the inter-electrode distance being shown against each trace. Each trace is composed of three superimposed sweeps (each the average of 100), so that the waveforms which are shown may be regarded as representative of $\mathbf{3 0 0}$ action potentials for each electrode arrangement.

From the top trace in Fig. 1, which shows the potential changes at the distal electrode relative to a remote electrode, it can be seen that the passage of a 
volley under a single electrode was accompanied by an initial downward (positive) deflection followed by a large upward (negative) deflection and a slow return to the baseline. From the second trace in Fig. 1 it can be seen that with both electrodes over the course of the nerve but $13 \mathrm{~cm}$. apart, the initial downward and upward deflections were unchanged, indicating the same sequence of positivity and negativity at the lower electrode as before. However, a second downgoing deflection appeared approximately $7 \mathrm{msec}$. after the stimulus, showing that the upper electrode then became negative with respect to the lower one. From the lower traces in Fig. 1 it is clear that as interelectrode distance was reduced, the peaks of the main upward deflection and of the second downward deflection fell progressively earlier; the amplitude of the initial downward deflection and of the main upward deflection also decreased.

These results may be explained on the assumption that the approach of a volley of nerve impulses to an electrode in a volume conductor is signalled by positivity which changes abruptly to negativity as impulses begin to pass under the electrode. According to Lorente de Nó (1947) an electrode near to a single fibre in a volume conductor would be expected to show a second positive deflection after the main negative deflection. However, this second positive deflection is not seen in the top trace in Fig. 1 and was not seen in previous monopolar recordings from human nerve (Gilliatt, Goodman, and Willison, 1961). This may be due to the fact that we are not dealing here with single impulses but with a volley, initially synchronous but followed by scattered impulses in more slowly conducting fibres. The contribution of these slower fibres might well explain the absence of a second positive deflection in our records.

If it is accepted that the contribution of a recording electrode is initially positive and then negative, the waveforms shown in Fig. 1 can be explained by the fact that negativity at the lower electrode causes an upward deflection, whereas negativity at the upper electrode, which is connected to the other side of the amplifier, must necessarily produce a deflection in the opposite direction. Thus the lower traces in Fig. 1 represent the sum of the potential changes from two electrodes activated in succession and connected to a differential amplifier.

It is easily proved that the waveform obtained from a pair of recording electrodes over a nerve is in fact the algebraic sum of the absolute values of the potential changes occurring at each electrode. In the experiment illustrated in Fig. 2 a pair of recording electrodes was placed over the median nerve at the elbow, the inter-electrode distance being $4 \mathrm{~cm}$. The stimulus was applied to the median nerve at the wrist,

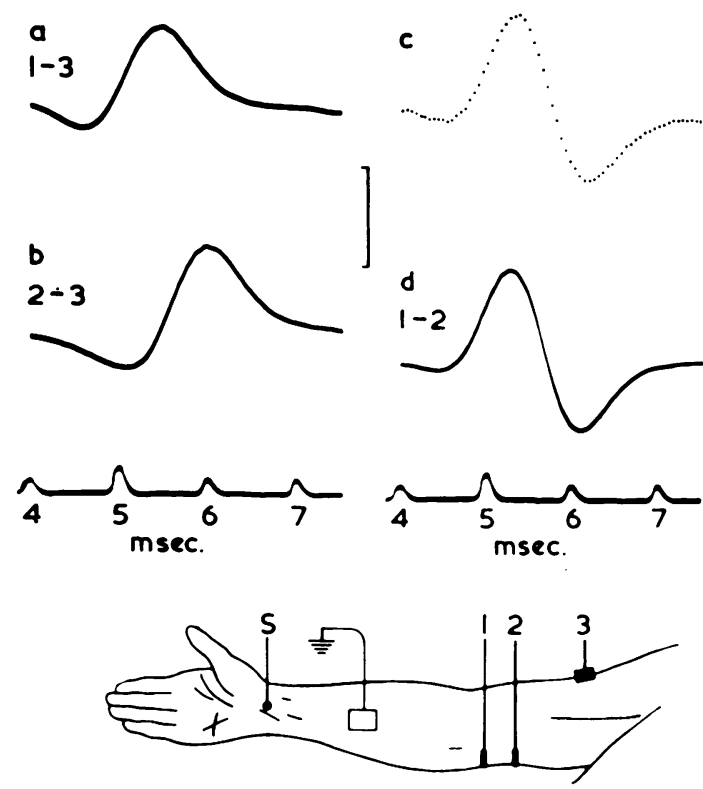

FIG. 2. Subject R.W.G. Action potentials from the median nerve with a pair of recording electrodes $4 \mathrm{~cm}$. apart. Stimulus unchanged throughout.

(a) Action potential recorded from electrodes 1 and 3.

(b) Action potential recorded from electrodes 2 and 3.

(c) Calculated action potential obtained by subtracting (b) from (a).

(d) Action potential recorded from electrodes 1 and 2 . Calibration $40 \mu \mathrm{V}$.

its intensity being unchanged throughout. Averaged records were made of the potential changes between the lower electrode of the pair and a remote electrode over the deltoid insertion (Fig. 2a), and between the upper electrode of the pair and the remote electrode (Fig. 2b). Further records were then made with the pair of electrodes connected to the amplifier, the result being shown in Figure 2d.

Figure $2 c$ is the calculated curve obtained by subtracting the waveform shown in Fig. $2 b$ from that shown in Figure 2a. Voltages at 100 points along each trace were measured from the photographic records, and the points composing the curve shown in Fig. $2 c$ were then obtained by subtraction. It can be seen that the waveform of this calculated curve is virtually identical with that of the recorded action potential shown in Figure 2d.

MEASUREMENT OF LATENCY If the onset of negativity at an electrode coincides with the passage of impulses under that electrode, it would seem reasonable to measure to this point when estimating conduction time for the fastest fibres contributing to the action potential. However, it has been suggested by some 


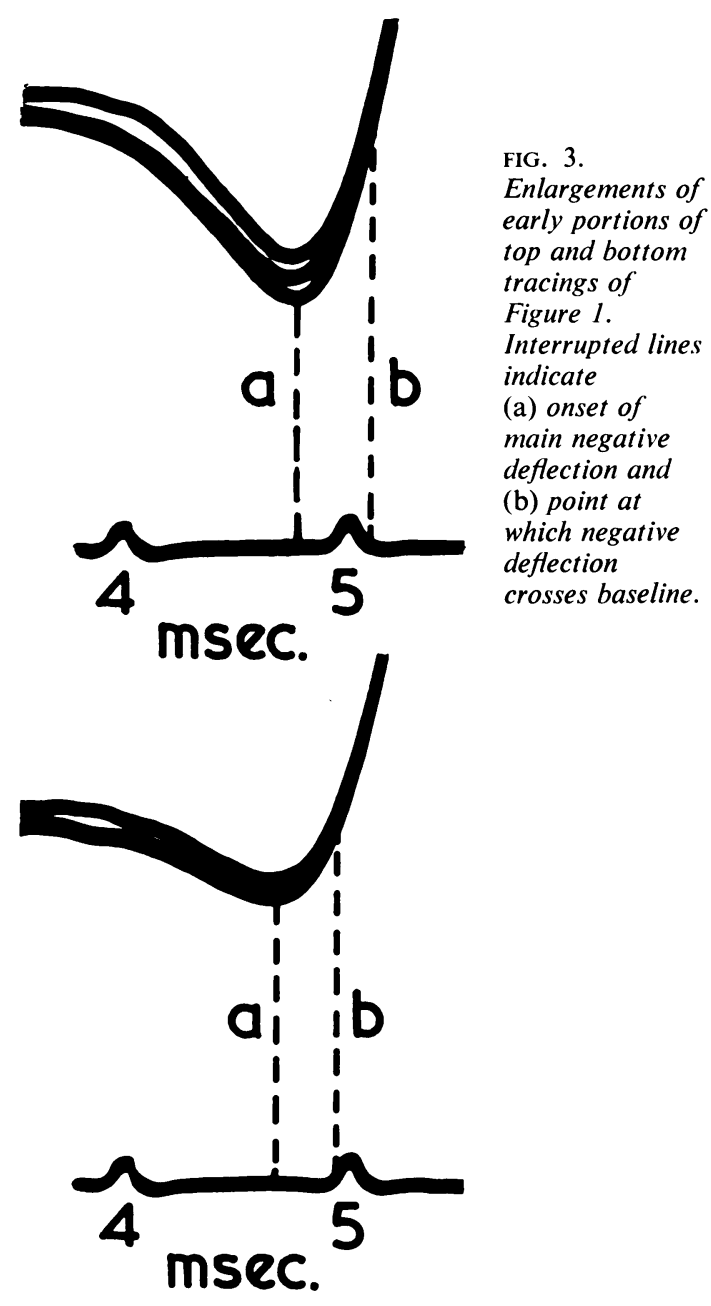

the onset of the negative deflection, but to the point at which this deflection crosses the baseline (Buchthal and Rosenfalck, 1965). While this may be correct in the case of monopolar recordings from single fibre preparations, the situation with regard to human nerve action potentials is more complex.

The difficulty may be illustrated by reference to Fig. 3 which shows the early parts of the top and bottom tracings of Fig. 1 in greater detail. The upper tracing in Fig. 3 is the monopolar recording and the lower tracing the record obtained with two electrodes over the nerve trunk and $3.0 \mathrm{~cm}$. apart. In the latter the initial downward deflection is small, presumably due to the fact that impulses approaching the lower electrode and causing positivity are already beginning to affect the upper electrode beyond it. The broken lines in Fig. 3 indicate $(a)$ the onset of the negative deflection and $(b)$ the point at which the negative deflection crosses the baseline, and it can be seen that the change in waveform between the upper and lower tracings has shifted the position of $b$ more than $a$. Thus, a latency measurement to point $a$ is less likely to vary according to the exact electrode arrangement than a measurement taken to point $b$.

All latency measurements given in subsequent sections of the paper have therefore been made to the onset of the negative deflection and not to the point at which the trace crosses the baseline.

MEASUREMENT OF CONDUCTION VELOCITY When conduction distance is divided by latency, a figure for conduction velocity is obtained. A calculation of velocity made in this way may involve certain errors. For example, any delay in the initiation of impulses under the cathode will be included in the latency, and although the excitation time for mammalian nerve is usually short (less than $0 \cdot 1 \mathrm{msec}$.), it is known from work with single fibres that longer delays may occur when the stimulus is at threshold for the fibre concerned (Erlanger and Gasser, 1937).

A second source of error is the possibility that current might spread from a cathode on the skin $\stackrel{0}{\overrightarrow{0}}$ and excite the nerve at a distance from it; excitation $\triangle$ could occur at a point between cathode and anode $\stackrel{\mathbb{Q}}{\varrho}$ or, alternatively, if strong shocks were used, currento might spread away from the cathode towards the recording electrodes, thus shortening the true conduction distance. For this reason most workers prefer to record from more than one point along the course of the nerve, and to estimate conduction velocity from the difference in the time taken for impulses to reach the two or more points from which recordings are made. In the case of human nerve action potentials, this involves, for example, stimulating sensory fibres in the finger and recording ascending impulses at the wrist and elbow in order to obtain a subtracted figure for conduction time between these points. However, no figures have been published to show whether this is in fact preferable to the simpler procedure of measuring latency directly from a cathode at the wrist to a recording electrode at or above the elbow. This problem has therefore been re-investigated.

EFFECT OF ANODE POSITION In four subjects a stimulating cathode $1.0 \mathrm{~cm}$. in diameter was placed over the ulnar nerve just above the wrist and three anode positions were selected, on $(a)$ the dorsum of the wrist, $(b)$ the fleshy part of the hypothenar eminence, and $(c)$ the base of the fifth finger. In the case of the finger the anode was a ring electrode, whereas at the other two sites either a pad electrode workers that measurement should be made, not to 


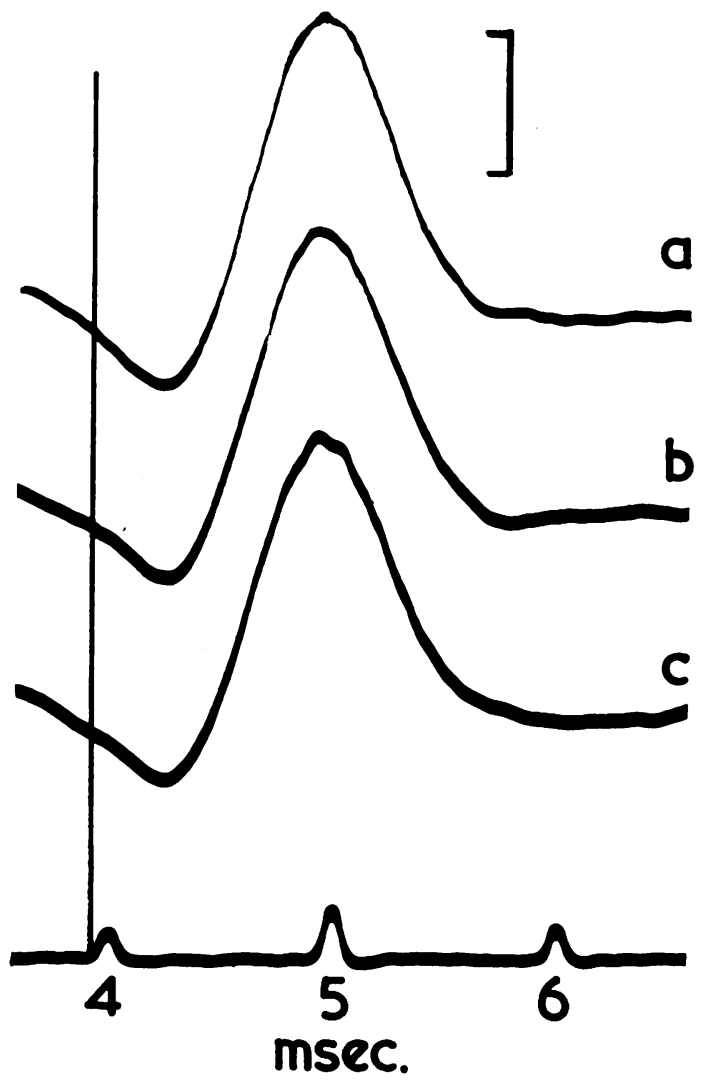

FIG. 4. Subject R.G.W. Action potentials from the ulnar nerve at the elbow; stimulating cathode over the nerve at the wrist. Anode placed (a) on dorsum of wrist, (b) over hypothenar eminence, and (c) at base of little finger. Stimulus intensity adjusted to give half-maximal responses. Calibration $5 \mu \mathrm{V}$.

or a metal plate was used. A pair of recording electrodes was placed over the course of the nerve above the elbow. Stimulus intensity was adjusted to give action potentials of half-maximal amplitude and the effect of different anode positions on latency was studied. One hundred responses were averaged for each anode position and the averaged responses photographed on the same frame to facilitate comparison.

Results were similar in all four subjects and are illustrated by the record shown in Fig. 4 from which it can be seen that action potential latency was unaffected by changes in anode position. This indicates that nerve impulses were in fact initiated close to the cathode and not at some point between cathode and anode.

The above conclusion requires qualification in one respect; when the anode as well as the cathode is close to the nerve trunk, excitation will occur at both electrodes. An example of this is illustrated in Fig. 5; in this experiment the cathode was placed over the median nerve at the wrist and the anode was either over the fleshy part of the thenar eminence (Fig. 5a), or at the base of the index finger (Fig. 5b). Recording electrodes were placed over the median nerve above the elbow, and the stimulus was adjusted to give an action potential of half-maximal amplitude. It can be seen from Fig. $5 b$ that when the anode was a ring electrode round the base of the index finger, anodal excitation of digital nerve fibres occurred, giving rise to a small late deflection in the record. This deflection could be abolished by increasing stimulus intensity, indicating that the same fibres were then excited through the cathode at the wrist.
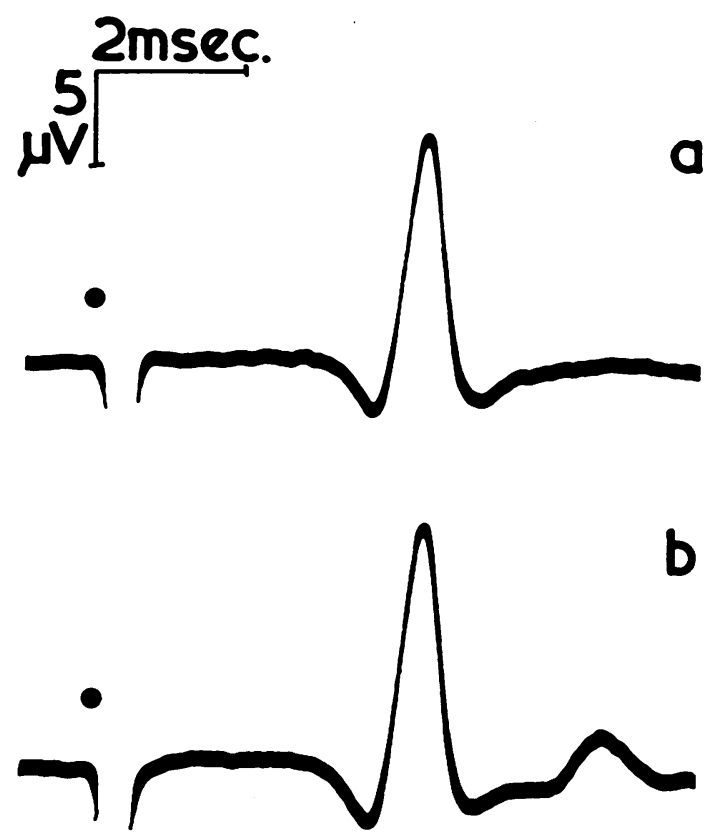

FIG. 5. Subject I.D.M. Action potentials from the median nerve at the elbow. Stimulating cathode over the nerve at the wrist. Anode placed (a) over thenar eminence and (b) at base of index finger. Stimulus intensity adjusted to give half-maximal response. The late deflection in (b) is caused by stimulation of digital fibres through the anode.

EFFECT OF STIMULUS INTENSITY The effect of stimulus intensity on action potential latency was investigated in six subjects. The stimulating cathode was placed over the median nerve at the wrist, the anode being a plate on the dorsum of the wrist; a pair of recording 
electrodes was placed over the median nerve above the elbow. For each subject, action potentials were first recorded with the stimulus voltage set just above the threshold value for the nerve, the action potentials which were recorded being approximately $5 \mu \mathrm{V}$ in amplitude. After three sets of 100 responses had been averaged and photographed, stimulus intensity was increased until the main negative deflection of the action potential ceased to grow in amplitude. This stimulus was considered to be maximal for those fibres in the nerve trunk which contributed to the main deflection of the potential. Stimulus voltage was then increased by a further $20-25 \%$ and another three sets of 100 responses were averaged and photographed. Results from the six subjects are shown in Table I, from which it can be seen that in each case the latency was shorter with a supramaximal than with a near-threshold stimulus. The difference in latency ranged from 0.05 to $0.15 \mathrm{msec}$., the mean difference being $0 \cdot 11 \mathrm{msec}$.

TABLE I EFFECT OF STIMULUS INTENSITY ON LATENCY

\begin{tabular}{lcc} 
Subject & \multicolumn{2}{c}{ Latency $($ msec.) } \\
\cline { 2 - 3 } & $\begin{array}{l}\text { Near-threshold } \\
\text { Stimulation }\end{array}$ & $\begin{array}{l}\text { Supramaximal } \\
\text { Stimulation }\end{array}$ \\
\hline M.D. & 3.70 & 3.60 \\
R.W.G. & 4.50 & 4.35 \\
A.L. & 3.65 & 3.50 \\
I.D.M. & 4.25 & 4.15 \\
R.G.W. & 4.70 & 4.65 \\
S.W. & 4.00 & 3.90 \\
\multicolumn{1}{c}{ Mean } & 4.13 & 4.02
\end{tabular}

An illustrative record from subject R.G.W. is shown in Figure 6. The action potential recorded during near-threshold stimulation is shown in Fig. 6a, its amplitude being $5.5 \mu \mathrm{V}$ and its latency $4.70 \mathrm{msec}$. In Fig. $6 \mathrm{~b}$ stimulus intensity was supramaximal, the amplitude of the action potential now being $35 \mu \mathrm{V}$ and its latency $4.65 \mathrm{msec}$. It can be seen that as a result of the high amplification used in Fig. 6a there is some irregularity of the averaged traces, which is not present at the lower amplification used to record the responses to supramaximal stimulation. This illustrates the value of superimposing several averaged traces when using high amplification; it allows accurate latency measurements to be made in spite of small random fluctuations of each trace.

COMPARISON OF WRIST AND FINGER STIMULATION In six subjects the digital nerve fibres in the middle finger were stimulated and action potentials recorded from the median nerve through two pairs of electrodes placed just above the wrist and elbow respectively. The median nerve was then stimulated
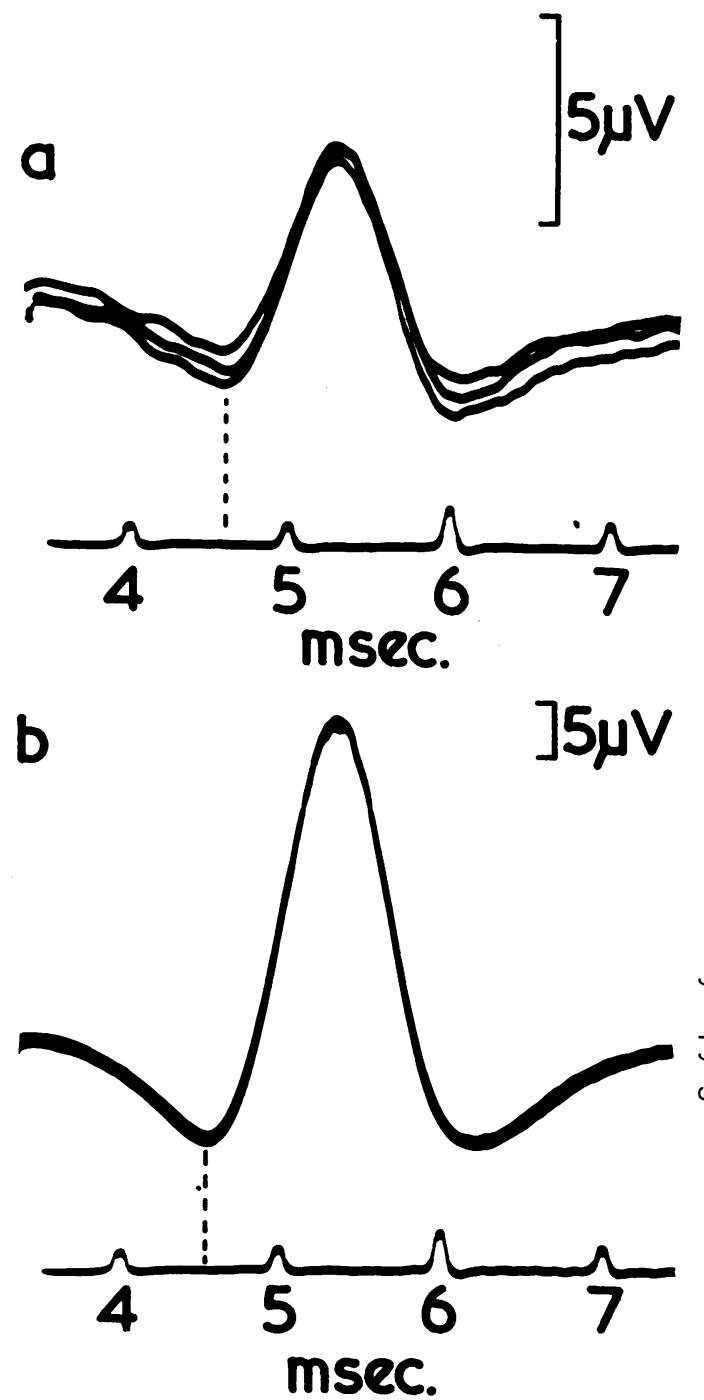

FIG. 6. Subject R.G.W. Action potentials from the median nerve at the elbow. Stimulus to the nerve at the wrist just above threshold in (a) and supramaximal in (b). Interrupted lines show latency of negative deflection in each case.

at the wrist through the distal electrode of the pair previously used for recording, and action potentials were again recorded at the elbow. The different electrode arrangements are shown in the lower part of Figure 7. From the records of finger stimulation a conduction time between wrist and elbow was obtained by subtraction; this was then compared with the figure obtained directly from wrist stimulation for conduction over the same distance. In making such a comparison it was obviously important to 

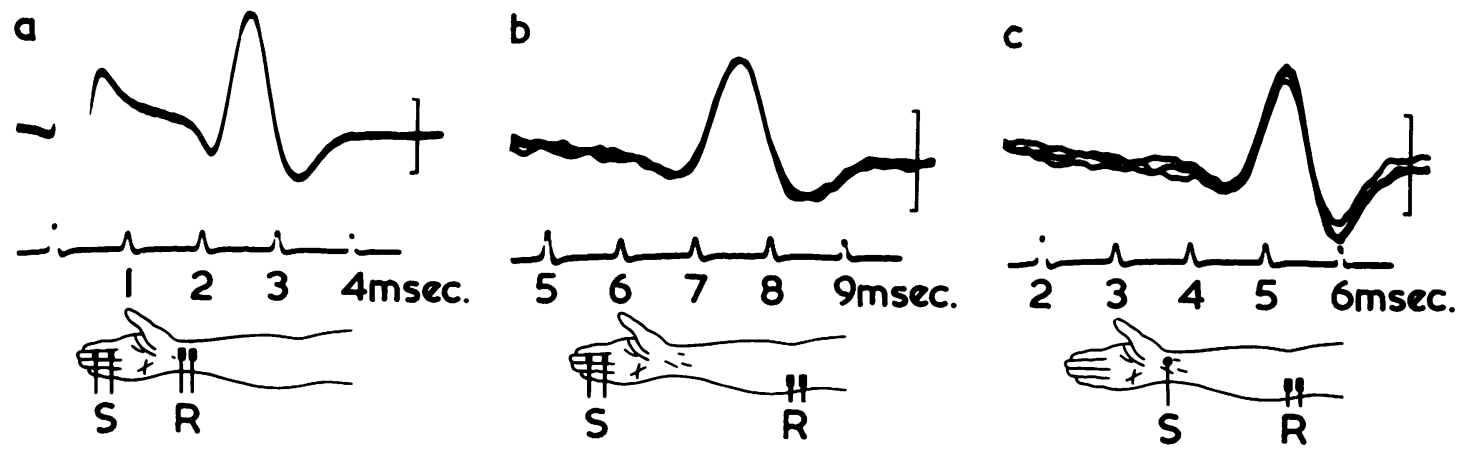

FIG. 7. Subject R.W.G. Action potentials from the median nerve to illustrate the estimation of conduction time between wrist and elbow by subtraction ((a) and (b)) and by direct measurement (c). Stimulating and recording sites shown below. Stimulus intensity unchanged between (a) and (b). Stimulus intensity in (c) below threshold for motor fibres to hand muscles. Calibration $20 \mu V$ in (a), $5 \mu V$ in (b) and (c).

ensure that the same nerve fibres were being stimulated at the two levels. For this reason the distal wrist electrode was placed over the median nerve at a point where a weak stimulus caused paraesthesiae to radiate into the middle finger. In all six subjects it was possible to produce paraesthesiae in the middle finger with a shock to the nerve at the wrist too weak to excite motor fibres to the thenar muscles.

Figure 7 contains illustrative records from subject R.W.G. Figure 7a shows the action potential recorded at the wrist. The action potential shown in Fig. $7 \mathrm{~b}$ was recorded at the elbow, the stimulus to the finger being unchanged. Figure $7 \mathrm{c}$ shows the action potential recorded at the elbow when the stimulus had been moved to the wrist and the shock strength reduced to avoid excitation of motor fibres. Results from six subjects are shown in Table II, from which it can be seen that there was no consistent difference between the conduction time obtained by subtraction and that measured directly. These results do not therefore suggest that a significant error is introduced if conduction time is estimated from a

\section{TABLE II}

CONDUCTION TIMES BETWEEN WRIST AND ELBOW IN THE MEDIAN NERVE OBTAINED BY SUBTRACTION AND BY DIRECT MEASUREMENT

\begin{tabular}{lcc} 
Subject & $\begin{array}{c}\text { Conduction Time by } \\
\text { Subtraction (msec.) }\end{array}$ & $\begin{array}{c}\text { Direct Latency } \\
\text { (msec.) }\end{array}$ \\
\hline M.D. & 3.25 & 3.30 \\
R.W.G. & 4.65 & 4.55 \\
A.H. & 3.45 & 3.45 \\
A.L. & 3.50 & 3.50 \\
I.D.M. & 3.95 & 3.95 \\
R.G.W. & 4.20 & 4.20 \\
\multicolumn{1}{c}{ Mean } & 3.83 & 3.82
\end{tabular}

single latency measurement rather than by subtraction.

In the case. of the ulnar nerve we were unable to stimulate digital fibres at the wrist without also exciting some motor fibres in the nerve trunk. The experiment described above was therefore slightly modified in order to answer a different question: if the shock to the wrist were strong enough to stimulate a substantial number of fibres passing to or from muscle, would these have a conduction velocity higher than that of digital fibres? The digital fibres of the fifth finger were therefore stimulated as before and action potentials recorded at the level of the wrist and elbow. The stimulus was then delivered through the distal electrode at the wrist, the shock strength being adjusted to give a brisk twitch of the ulnarsupplied hand muscles, and nerve action potentials were again recorded at the level of the elbow. The results of this procedure in six subjects are shown in Table III, from which it can be seen that the direct latency measurement was in every case slightly shorter than the conduction time obtained by subtraction, the difference ranging from $0.05 \mathrm{msec}$. to

\section{TABLE III}

CONDUCTION TIMES BETWEEN WRIST AND ELBOW IN THE ULNAR NERVE OBTAINED BY SUBTRACTION AND BY DIRECT MEASUREMENT

\begin{tabular}{lcc} 
Subject & $\begin{array}{c}\text { Conduction Time by } \\
\text { Subtraction }(\text { msec.) }\end{array}$ & $\begin{array}{c}\text { Direct Latency } \\
(\text { msec. })\end{array}$ \\
\hline M.D. & 4.35 & 4.30 \\
R.W.G. & 5.30 & 5.20 \\
A.H. & 3.95 & 3.90 \\
A.L. & $4 \cdot 25$ & $4 \cdot 15$ \\
I.D.M. & 4.60 & 4.55 \\
R.G.W. & 5.40 & 5.35 \\
$\quad$ Mean & 4.64 & 4.57
\end{tabular}


0.10 msec. with a mean of 0.07 msec. This difference is small in relation to the actual conduction time and even if it were wholly due to a difference in conduction velocity between fibres in muscular and digital branches of the ulnar nerve, this difference would only be of the order of 1 to $2 \%$. In fact the differences shown in Table III may well be due to the stronger shocks used for wrist stimulation, since it has already been shown that increased shock strength has the effect of reducing latency by at least this amount (see Table I). From these experiments it may therefore be concluded that if there are fibres in the muscle branches of the ulnar nerve with a substantially higher conduction velocity than digital fibres, they are not present in sufficient numbers to affect the action potentials which we have recorded.

\section{DISCUSSION}

Now that the recording of nerve action potentials is a commonplace clinical procedure, it may seem surprising that little attention has been paid to such a basic problem as the most suitable arrangement of recording electrodes. From our results it is clear that the waveforms recorded from a single active electrode are simpler to interpret than those obtained when the nerve volley passes successively under two electrodes placed a short distance apart over the nerve trunk. On the other hand, the latter arrangement offers much better rejection of random activity derived from muscle in subjects who do not relax well during the procedure. This is obviously an important factor when dealing with apprehensive patients rather than with trained subjects. If, however, a pair of active electrodes is used, the interelectrode distance will affect both the amplitude and duration of the recorded potential; thus published figures for the range of normal are only helpful to other workers when the inter-electrode distance is also given.

Even when the inter-electrode distance is standardized, there is a further complication derived from the fact that the recorded potential depends on the contribution of two electrodes. If one electrode is closer to the nerve than the other its contribution will be increased so that mainly up-going or mainly down-going potentials may be recorded from the same nerve merely due to lateral displacement of one electrode. Thus, in the case of pathological nerve it is usually wise to check an abnormal waveform by monopolar recording before accepting that it is due to changes in the nerve itself.

The extent to which current spreads from under a stimulating electrode on the skin is important when either motor or sensory fibres are being examined. Henriksen (1956) considered that stimulation occurred under the centre of a circular cathode on the skin regardless of the size of the cathode. In contrast $\mathbb{D}$ to this, Pinelli (1964) found that the latency of hypothenar muscle action potentials might be of shortened by as much as $0.75 \mathrm{msec}$. if a stimulus to $\overrightarrow{0}$ the ulnar nerve at the elbow was increased from 0 maximal to four times maximal; he considered that this was due to current spread. However, when action potentials from muscle rather than nerve are re- $\stackrel{0}{\overrightarrow{2}}$ corded, the situation is complicated by the fact that supramaximal stimuli may spread to adjacent nerves and thus excite muscles at a distance from the $\frac{\vec{\sigma}}{0}$ recording electrodes, their potentials tending to cause early deflections on the trace (Gassel, 1964). Our $\frac{}{\sigma}$ own findings do not in fact suggest that current $\vec{\Phi}$ spread from under a cathode introduces a serious $\stackrel{\varrho}{\Omega}$ error in estimations on normal nerve, provided that $क$ stimulus intensity is not more than $25 \%$ supramaxi- $\overrightarrow{0}$ mal, but this should not be taken to mean that the $\vec{\overrightarrow{ }}$ same is true of pathological cases in which the $\vec{\omega}$ electrical threshold of affected nerve fibres may be raised. When very strong stimuli are needed to excite $\frac{5}{0}$ pathological fibres current spread could become a i serious problem and more studies are needed on this point. For example, it may be that in such cases the cathode should not be a pad on the skin but $\$$ needle inserted close to the nerve as described by Gassel and Trojaborg (1964).

It is often asked whether an adequate estimate of $\bar{c}$ conduction velocity can be obtained from the time of conduction of an impulse from a stimulatin cathode to a single recording site, or whether a series of of recording electrodes should be placed along the course of the nerve. Our own results do not suggest that the former method introduces a significant error, but reservations must again be made about the effects of very strong shocks if these are required to excite pathological nerve.

In his paper describing stimulation of digital $\overrightarrow{\vec{P}}$ fibres in the fingers and the recording of nerve action $\frac{3}{3}$ potentials at the level of the wrist and elbow, Dawson (1956) compared conduction times between wrist and elbow for ascending volleys from the fingers and for motor volleys to the small muscles of the hand. When measured over the same length of nerve $:$ in the same experiment, conduction velocity in sensory 3 fibres from the fingers was usually slightly greater than that of the fastest motor fibres, and Dawson com- $\frac{3}{3}$ mented '. . . it is therefore probable that the finger afferents are larger than the motor fibres and as large as muscle afferents'.

In our own experiments stimulation of the ulnar nerve at the wrist did not give rise to an ascending o volley with a conduction velocity significantly higher $N$ than that obtained from stimulating digital fibres in the fingers, although the shock at the wrist was 
sufficient to produce brisk twitches of the ulnarsupplied hand muscles. Such a shock would be expected to excite a substantial number of muscle afferent fibres, so that our findings support Dawson's suggestion that finger afferents have a conduction velocity, and therefore a fibre diameter, similar to that of muscle afferents.

One final point requires discussion. In the present experiments a barrier grid storage tube has been used to obtain averaged records. Other averaging methods are possible, such as that used in the Computer of Average Transients (CAT) developed by the Mnemotron Corporation, and further work is needed to assess the relative merits of the two systems. However, it is clear from the present work that an averaging technique has great advantages over the older method of photographic superimposition; latency measurements with the accuracy described here could not have been obtained from photographic records of the primary trace.

One criticism which can be made of averaged records is that any physiological variation in the response, which might occur between one sweep and another, is eliminated by the averaging process. This criticism has particular relevance to averaged records from the brain, since cerebral evoked responses may vary in amplitude according to the amount of spontaneous activity (Abrahams, Langworth, and McDonald, 1963). When recording peripheral nerve action potentials, however, there is no evidence of significant variation in either the amplitude or the latency of successive responses. It might be argued that some variation in latency would be expected from the work of Erlanger and Gasser (1937) who showed in single nerve fibres that there is a delay in the initiation of a nerve impulse when the stimulus is near threshold. However, this does not appear to be of significance when the compound action potential derived from many fibres is recorded through the skin; in these circumstances nearthreshold stimuli do not cause a detectable variation in response latency when compared with supramaximal stimuli (see Fig. 8 of Gilliatt and Willison, 1963). Thus we conclude that when recording from peripheral nerves, an averaging process may safely be used to eliminate noise and random activity without obscuring details of individual action potentials.

\section{SUMMARY}

A response averager incorporating a barrier grid storage tube was used to study action potentials recorded from the median and ulnar nerves of healthy subjects.

The waveforms obtained by monopolar and bipolar recording were compared, and the relative advantages of the two methods are discussed.

The spread of current from a cathode on the skin was studied. With weak stimuli to the median nerve at the wrist, the site of excitation was under the cathode, but increasing stimulus intensity from nearthreshold to $25 \%$ supramaximal shortened action potential latency by 0.05 to $0.15 \mathrm{msec}$. in different subjects, suggesting some current spread. Latency was unaffected by anode position.

When the ulnar nerve at the wrist was stimulated by a shock sufficient to excite branches to the hand muscles, the velocity of the ascending action potential was not significantly higher than that of a volley produced by stimulation of digital nerve fibres in the fifth finger. From this it is concluded that few if any of the muscle afferent fibres in the ulnar nerve at the wrist have a diameter or conduction velocity greater than that of digital afferent fibres.

We are indebted to Mr. H. B. Morton for helpful discussions and advice during the construction of the response averager, and to the Central Research Fund of the University of London for a grant to cover the cost of components. We also wish to thank Dr. W. A. Cobb for allowing us to use facilities in the Department of Applied Electrophysiology.

\section{APPENDIX}

THE RESPONSE AVERAGER

The problem to be solved by an averaging technique is the separation of an event, e.g., the response to a stimulus, from random activity of greater amplitude, which obscures it. This random activity may be of high frequency (amplifier noise) or low frequency (background muscle activity) but in either case its voltage is such that the response under investigation is obscured. Separation of the wanted signal is made possible by the fact that it occurs at a fixed point in each sweep whereas the unwanted activity occurs irregularly. If, therefore, the amplitudes of successive traces are summed algebraically at a series of intervals following a stimulus, the response to that stimulus becomes proportionately larger as irregular activity tends to cancel out.

Thus an automatic summation or averaging technique, such as that described by Dawson (1954), involves storing a series of sweeps in such a way that potentials at fixed intervals during the sweep may be summed. After summation, a single sweep composed of the summed potentials for each interval is then recovered. This, when suitably calibrated, represents the average of all the sweeps stored. In Dawson's electro-mechanical averager it was possible to sum potentials at only 62 points on a $100 \mathrm{msec}$. timescale, and while this was adequate for the relatively slow potential changes which may be recorded from the brain, it made the apparatus unsuitable for work with peripheral nerves, in which the waveforms to be delineated have durations of the order of 2-3 msec. 
The barrier grid storage tube (Jensen, 1955; Gibbons, 1961 ; Paul, 1962) was originally designed for use in radar apparatus. Methods of using the E.M.I. 9511A tube for averaging electro-physiological responses were developed by Buller and Styles (1959) and by Cooper and Warren (1961). The type 9511A is an electronic charge-storage tube containing a dielectric sheet storage surface backed by a conducting plate and faced by a fine mesh, termed the 'barrier grid'. The scanning beam, which is electrostatically focused and deflected, passes through the barrier grid and deposits charge on the storage surface. The presence of a conducting grid of fine mesh close to the target dielectric permits the writing-in of both positive and negative charges. A single electron gun is used for both storing and reading. While it is possible to obtain about 20 consecutive readings of a stored signal, the best results are obtained if one reading process erases the charge pattern; in this case it is necessary to photograph the single output waveform on a monitor oscilloscope. Since overloading of the storage tube results in loss of linearity, another monitor tube is necessary to ensure that the correct input voltage is not exceeded.

When the storage tube is being used to average a number of evoked responses, the time base is triggered with a constant time relationship to the stimulus, and the beam moves across the top of a rectangular area on the target. During the flyback at the end of the sweep, the beam is stepped downwards, so that when next triggered, it moves across the target beneath the path of the first trace. This allows up to 100 sweeps to be stacked vertically. So that the target is fully utilized whatever the number of sweeps stored, spot-wobble is added to each writing sweep, broadening the lines in the correct proportion so that they touch. When the appropriate number of sweeps has been stored, the beam reaches the bottom of the inscribed area, and the scanning process stops. The charge analogues of parts of the responses having constant latency with respect to the stimulus are now aligned vertically across the target.

To average these responses, the beam current is increased a hundredfold, and the beam is swept across the target once more, but with a spot-wobble large enough to cover the whole charged area. The frequency of the spotwobble is $100 \mathrm{Kc} / \mathrm{sec}$., which is much higher than any expected signal component. In discharging the target by this method, the read amplifier is provided with 200 samples every 10 microseconds, and being unable to follow this rapid sampling frequency, it responds only to the mean amplitude, thus fulfilling the requirements of an averager.

The definition is a function of spot size, and for the E.M.I. $9511 \mathrm{~A}$ it is equivalent to 150 lines of a televisiontype raster. This implies a limitation of the high frequency response, although the absolute value of the cutoff frequency is proportional to the sweep speed. The calibration tracing in Fig. 1 illustrates the high frequency loss imposed by the averager at the sweep speed used in the present experiments.

\section{REFERENCES}

Abrahams, V. C., Langworth, E. P., and McDonald, M. (1963). Interaction between evoked potentials and background electrical activity in the brain stem of the cat. Its effect on 'computer averaging'. J. Physiol. (Lond.), 168, 12-13P.

Buchthal, F., and Rosenfalck, A. (1965). Action potentials from sensory nerve in man; physiology and clinical application. Acta neurol. scand., in the press.

Buller, A. J., and Styles, P. R. (1959). A new averaging technique for improving the signal-to-noise ratio of evoked potentials. J. Physiol. (Lond.), 149, 65P.

- 1 (1961). Improvement in signal/noise ratio with the aid of a barrier grid storage tube. In Proc. 3rd int. Confr. on Medical Electronics, London 1960, pp. 63-64. Institution of Electrical Engineers, London.

Campbell, E. D. R. (1962). The carpal tunnel syndrome: investigation and assessment of treatment. Proc. roy. Soc. Med., 55, 401-405.

Cooper, R., and Warren, W. J. (1961). Use of a barrier grid storage tube for averaging evoked responses. Electroenceph. clin. Neurophysiol., 13, 141-142.

Dawson, G. D. (1954). A summation technique for the detection of small evoked potentials. Ibid., 6, 65-84.

- (1956). The relative excitability and conduction velocity of sensory and motor nerve fibres in man. J. Physiol. (Lond.),

131, 436-451. through skin in man. J. Neurol. Neurosurg. Psychiat., 12, $\omega$ 259-267.

Downie, A. W., and Newell, D. J. (1961). Sensory nerve conductio $\overrightarrow{0}$ in patients with diabetes mellitus and controls. Neurolog (Minneap.), 11, 876-882.

Erlanger, J., and Gasser, H. S. (1937). Electrical Signs of Nervous Activity. University of Pennsylvania Press, Philadelphia.

Gassel, M. M. (1964). Sources of error in motor nerve conduction studies. Neurology (Minneap.), 14, 825-835.

Gassel, M. M., and Trojaborg, W. (1964). Clinical and electre physiological study of the pattern of conduction times in thes. distribution of the sciatic nerve.J. Neurol. Neurosurg. Psychiain 27, 351-357.

Gibbons, D. J. (1961). The barrier grid storage tube. Electronic Eng. 33, 630-636.

Gilliatt, R. W., Goodman, H. V., and Willison, R. G. (1961). The recording of lateral popliteal nerve action potentials in man. J. Neurol. Neurosurg. Psychiat., 24, 305-318.

- and Willison, R. G. (1963). The refractory and supernormal periods of the human median nerve. Ibid., 26, 136-147.

patients with peripheral nerve lesions. Ibid., 21, 109-118.

Henriksen, J. D. (1956). Conduction velocity of motor nerves in normal subjects and patients with neuromuscular disorders. M.S. (Phys. Med.) Thesis. University of Minnesota.

Jensen, A. S. (1955). The radechon, a barrier grid storage tube. Radio Corporation of America Review, 16, 197-215.

Lorente de Nó, R. (1947). A Study of Nerve Physiology, part 2. Stud. Rockefeller Inst. med. Res., 132, 384-482.

Paul, R. J. (1962). The barrier grid storage tube and its use in integration. Brit. Comm. Electronics, 9, 28-32.

Pinelli, P. (1964). Physical, anatomical and physiological factors in the latency measurement of the $M$ response. Electroenceph. clin. Neurophysiol., 17, 86.

Pitman, J. R. (1958). A digital delay and time-scale generator. J. Physiol. (Lond.), 142, 3-5P.

Sears, T. A. (1959). Action potentials evoked in digital nerves by stimulation of mechanoreceptors in the human finger. Ibid. 148, 30-31P. 